This item was submitted to Loughborough's Research Repository by the author.

Items in Figshare are protected by copyright, with all rights reserved, unless otherwise indicated.

\title{
Productionisation issues for commercialisation of microfluidic based devices
}

PLEASE CITE THE PUBLISHED VERSION

PUBLISHER

(C) Emerald

VERSION

AM (Accepted Manuscript)

LICENCE

CC BY-NC-ND 4.0

REPOSITORY RECORD

Webb, D. Patrick, Benedikt J. Knauf, Changqing Liu, David A. Hutt, and Paul P. Conway. 2019. "Productionisation Issues for Commercialisation of Microfluidic Based Devices". figshare. https://hdl.handle.net/2134/5487. 
This item was submitted to Loughborough's Institutional Repository (https://dspace.lboro.ac.uk/) by the author and is made available under the following Creative Commons Licence conditions.

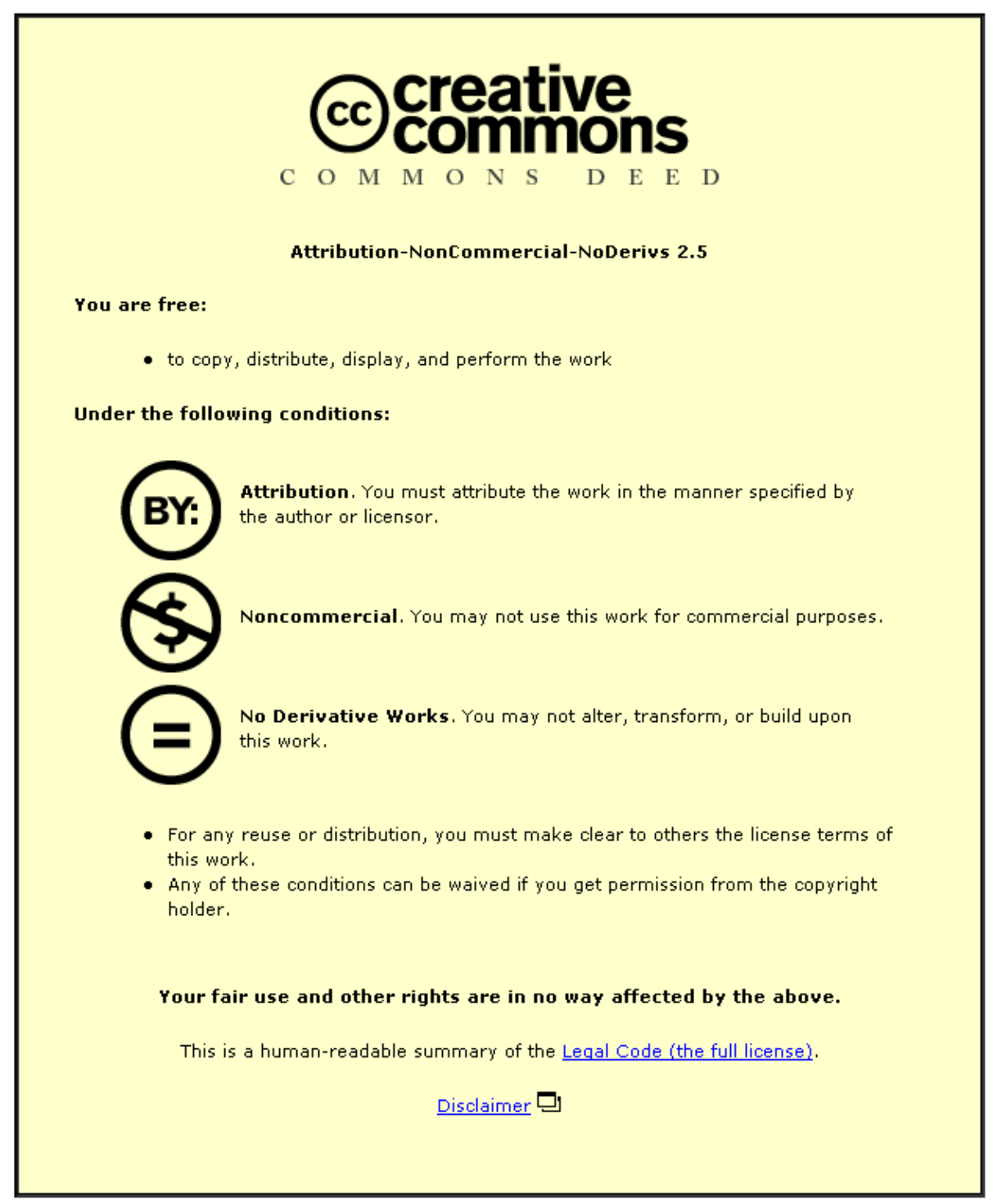

For the full text of this licence, please go to: http://creativecommons.org/licenses/by-nc-nd/2.5/ 


\title{
Productionisation Issues for Commercialisation of Microfluidic Based Devices
}

\author{
D.P.Webb, B.J.Knauf, C.Liu, D.A.Hutt and P.P.Conway \\ School of Mechanical and Manufacturing Engineering \\ Loughborough University \\ Loughborough LE11 3TU
}

\begin{abstract}
Purpose

Microfluidic or "lab-on-a-chip" technology is seen as a key enabler in the rapidly expanding market for medical point-of-care (POC) and other kinds of portable diagnostic device. In this paper we discuss two proposed packaging processes for large scale manufacture of microfluidic systems.

Design/methodology/approach

In the first packaging process, polymer overmoulding of a microfluidic chip is used to form a fluidic manifold integrated with the device in a single step. The anticipated advantages of the proposed method of packaging are ease of assembly and low part count. The second process involves the use of low frequency induction heating (LFIH) for the sealing of polymer microfluidics. The method requires no chamber, and provides fast and selective heating to the interface to be joined.
\end{abstract}

Findings

Initial work with glass microfluidics has demonstrated feasibility for overmoulding through two separate sealing principles. One is using the overmould as a physical support structure and providing sealing using a compliant ferrule. The other relies on adhesion between the material of the overmould and the microfluidic device to provide a seal. As regards LFIH work on selection and structuring of susceptor materials is reported, together with analysis of the dimensions of the heat affected zone. Acrylic plates have been joined using a thin $(<10 \mu \mathrm{m})$ nickel susceptor providing a fluid seal that withstood a pressure of $590 \mathrm{kPa}$.

Originality/value

Microfluidic chips have until now been produced in relatively small numbers. To scale-up from laboratory systems to the production volumes required for mass markets, packaging methods need to be adapted to mass manufacture.

\section{Introduction to Microfluidics}

Microfluidics is the technology of handling micro-litre to pico-litre volumes of fluids. Fluids flow through micron-sized channels formed in the surface of silicon, glass or polymer substrates. Fabrication is by lithography for silicon or glass, and by moulding or forming techniques for polymers. The simplest devices rely entirely on capillary forces to drive flow, while more complex devices incorporate electrodes for electrokinetically driven flow, and for electrical sensing of chemical species. Moving parts such as pumps and valves are still relatively rare (Verpoorte and De Rooij 2003). 
Microfluidic technology allows the miniaturisation and integration of chemical and biochemical laboratory processes. The two major classes of device are lab-on-a-chip (LOC), in which multiple chemical or biochemical operations are integrated on a single substrate, and micro-arrays(Schena 2000), supporting multiple, parallel identical or similar reactions. The advantages of miniaturised fluid handling in these applications include faster reaction times, integration of multiple functions with reduced sample handling, improved reaction control, inherent scalability of processes (scale up by scale out), reduced reagent and sample consumption and hence lower cost, and the ability to make portable systems. Finally making microfluidic devices by mass manufacture, batch-processing techniques yields the advantages of low cost and high product consistency.

Miniaturisation and the advantages outlined above make possible systems to carry out analysis close to the point of sampling. This capability services the growing trend to delivering healthcare in the near-patient or point-of-care (POC) setting (Sia SK and Kricka LJ 2008). Microfluidic devices have also addressed the need for high sample throughput capability generated by genetic mapping projects such as the Human Genome Project (Hupert et al. 2003), and by the mass screening by pharmaceutical companies of their large libraries of pharmaceutical compounds to find potentially druggable targets (Freemantle 1999). Other applications are chemical and biochemical synthesis in microfluidic reactor systems, and agricultural and environmental monitoring. Microfluidic technology will play an important role as a micro-to-nano interface in nanotechnology products. An example is a blood glucose sensor using carbon nanotubes as the sensitive element, deposited on microstructured electrodes(Kalaugher 2004). In addition fluid flows on the microscale can be engineered to control the motion of molecules individually (Ho 2001).

\section{Barriers to Commercialisation of Microfluidic Based Products}

Microfluidics has been a defined research area(Verpoorte and De Rooij 2003) for around 20 years since the development of micro-total analysis systems ( $\mu$ TAS) (Manz, Graber, and Widmer 1990). The field is now in the early stages of commercialisation with high growth rates predicted (Mounier and Provence 2003). However up to now most microfluidic chips have been produced singly or in small batches in laboratories so both manufacturing techniques and design are in need of productionisation.

Figure 1. A microfluidic assembly consisting of a fluidic substrate containing the microfluidic channels, a capping layer sealing the channels, fluidic input/output ports and a structure to support fluidic interconnection such as connectors for tubing.

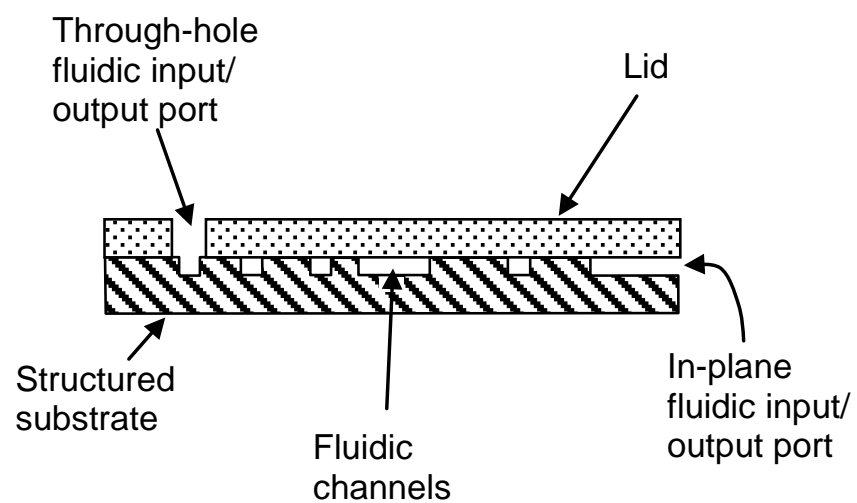


A typical microfluidic assembly might be as depicted in Figure 1(although there are many variations, for example both the substrate and lid may be structured). Techniques for creating the microfluidic channel network in the fluidic substrate are mostly borrowed from the silicon processing (photolithography and micromachining (PRIME Faraday Technology Watch 2002)) or plastics processing (embossing, injection-moulding) (Becker and Gaertner 2008) sectors and are inherently suited to large production volumes. Techniques used for packaging and integration are more problematic. Fluid interconnection in particular has been recognised for some time as a key area for increased research effort (Verpoorte and De Rooij 2003). Improved methods of bonding the lid to the fluidic channel layer are also required. A useful comparison is with the development of the micro-electromechanical systems (MEMS) sector where packaging has been found to be a major barrier between concept and market. It is estimated that up to $90 \%$ of the development costs of a new device are in the packaging (PRIME Faraday Technology Watch 2002). The pattern established in MEMS has been of development for applications that justify the initial costs, followed by exploitation of the product in other markets. For example accelerometers, originally developed for automotive safety, are now cheap enough to be incorporated in consumer electronics (Bourne 2005).

Current commercial microfluidic based diagnostic systems such as i-STAT (www.abbottpointofcare.com), consist of a disposable cartridge that plugs into a reader. The sample, for example whole blood, is driven through a passive microfluidic network by capillary forces so that packaging requirements are limited to assembly of the cartridge and electrical connections to the reader. Future applications will involve assays of increasing complexity (Sia SK and Kricka LJ 2008) so that interconnection requirements will include support for pressure driven flow (Ellis 2009), and for methods of integrating multiple miniaturised fluidic elements together. These may include sample preparation stages and microfluidic networks fabricated in different substrates. Work on the development of methods for interconnection and sealing of microfluidic systems at Loughborough University, which are suitable for manufacturing scale-up, are reviewed and updated in the sections below.

Other problems for commercialisation of microfluidic based devices concern the use of polymers. While polymers are the lowest cost option for large scale manufacturing, functional prototyping in polymers is a problem. Polymer manufacture is low cost in high production volumes because of the cost of the tooling. Thus small batches of microfluidic devices are cheaper to make in other materials such as glass. However a glass prototype may not deliver convincing proof-ofconcept because fluid flow is dominated by interactions with microchannel walls, and the wetting properties of glass are very different to those of polymers. Another reported problem is interactions of biomolecules with polymers which are not well documented. In general because polymers are less inert than glass compatibility issues must be considered(Hupert et al. 2003). 


\section{Thermoplastic Overmoulding for Modularisation and Integration of Microfluidic Devices}

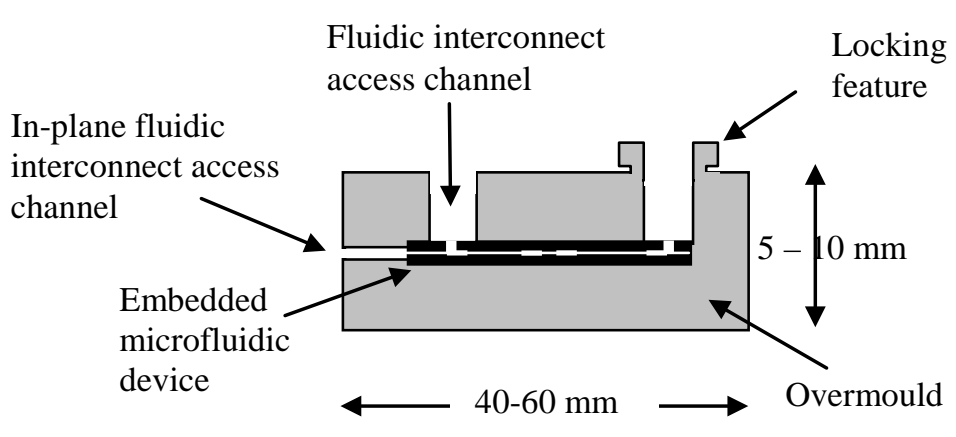

Figure 2. Concept of moulded interconnect to an embedded microfluidic device.

A method of packaging microfluidic devices to support fluidic interconnects by insert moulding has been proposed (Webb et al 2009). The concept is illustrated in figure 2. The microfluidic device is embedded in a polymer overmould by injection moulding or other plastics moulding technique. During overmoulding, pins within the mould tool are used to create fluidic access channels to the microfluidic device input/output ports. The access channels are several millimetres long and provide the basis for engineering of robust connections to tubes, or other means of fluidic interconnect, by addition of structures to the moulding. A microfluidic chip is thus converted from a stand-alone device into a module that can be integrated into a larger system. The method is intended to be compatible with mass manufacture, featuring use of high throughput processes (injection moulding), low part count and ease of assembly.

Proof of concept studies have been published elsewhere (Webb et al 2006, 2009) for an implementation where the fluid seal between the overmould and the microfluidic device relies on direct adhesion. That is to say that the moulding material forms an adhesive bond to the material of the insert on solidification. While insert moulding as a technology usually relies on mechanical interlocking for part integrity, the phenomenon of direct adhesion of moulding resins to metals has been reported in the literature, for example by Grujicic et al (Grujicic et al. 2008) for automotive parts. Direct adhesion to glass has not been previously reported. In the proof of concept studies the fluid seal produced by the adhesion of several injection moulding grade commercial resins (PA12, LCP, PPS, COC, ABS) to glass was studied. The polyamide was found to be the best performer. In the most recent work the fluid seal between polyamide 12 and glass was demonstrated to survive pressurisation with water at 1.4 bar (20 psi) at room temperature for 116 hours. Current work focuses on reducing the variability in the performance observed and selection of adhesion promotion coatings. 


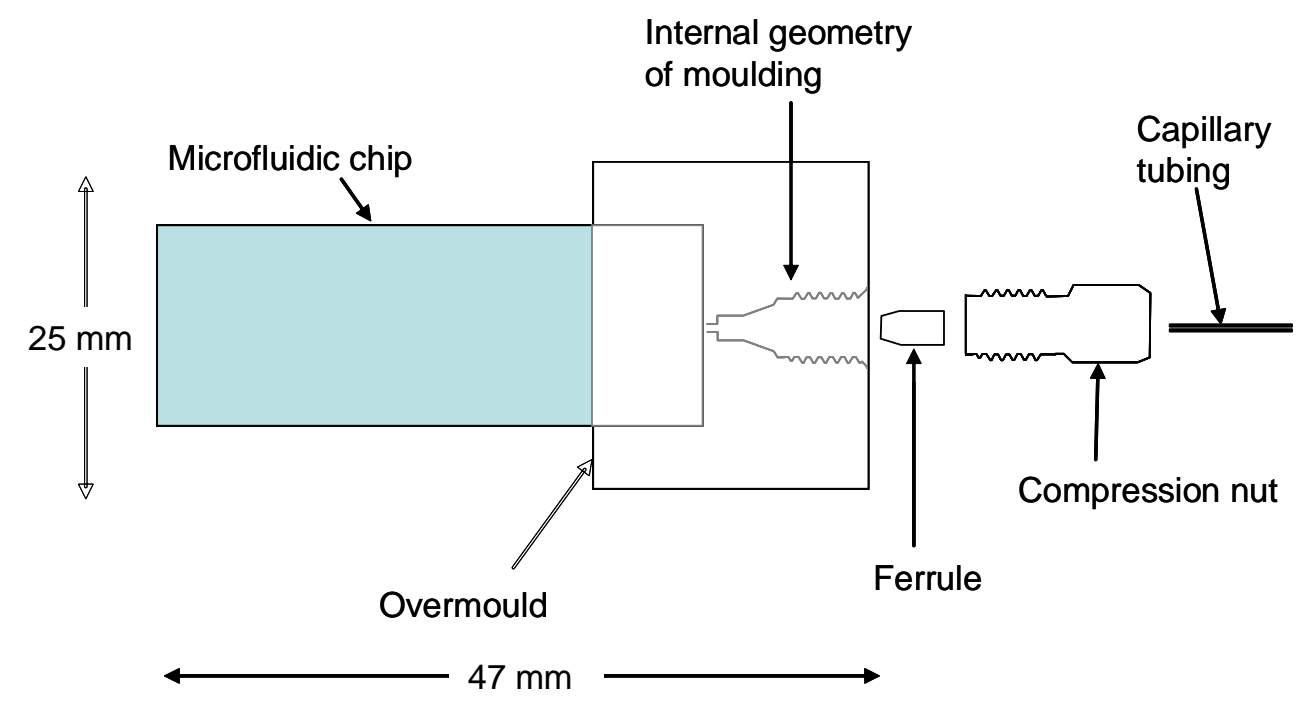

Figure 3. Design of moulding to allow connection of a capillary tube to a microfluidic device using a screw thread compression fitting.

A second approach is to introduce a ferrule for sealing and to use only the mechanical properties of the moulding to support an interconnect. Test mouldings embodying this concept were manufactured in polypropylene according to the design shown in Figure 3 to connect a microfluidic device with in-plane fluidic i/o ports to capillary tubing. The design features a thread and interior dimensions designed to fit an Upchurch 10/32 Nanotight female compression fitting. The fitting consists of a PEEK compression nut and a perfluoroelastomer ferrule as shown in Figure 3 Upchurch Scientific (www.idex-hs.com) is a brand for a range of high pressure fittings for connection of capillary tubing. These include Nanoport, the only commercial offering of a generic connector for microfluidic chips, which is also made from PEEK. In designing the tool for the overmould there was some concern that the much lower stiffness of polypropylene compared to PEEK would mean that the ferrule would not be compressed sufficiently in use to ensure a fluid good seal. The overall thickness of the overmould was therefore set at $7 \mathrm{~mm}$, allowing a high wall thickness of $1.5 \mathrm{~mm}$ in the region of the screw thread.

A finished moulding with a glass microfluidic chip insert is shown in Figure 4. The capillary was 1/16 inch outer diameter Teflon tubing. Water was observed to flow through the chip and emerge at the far end when the assembly was pressurised. Three mouldings using glass blanks instead of a microfluidic chip were pressure tested with water at 6 bar for 5 hours. A red dye was added to the water and the assemblies visually inspected at intervals for leaks. No leak was observed during the test. 


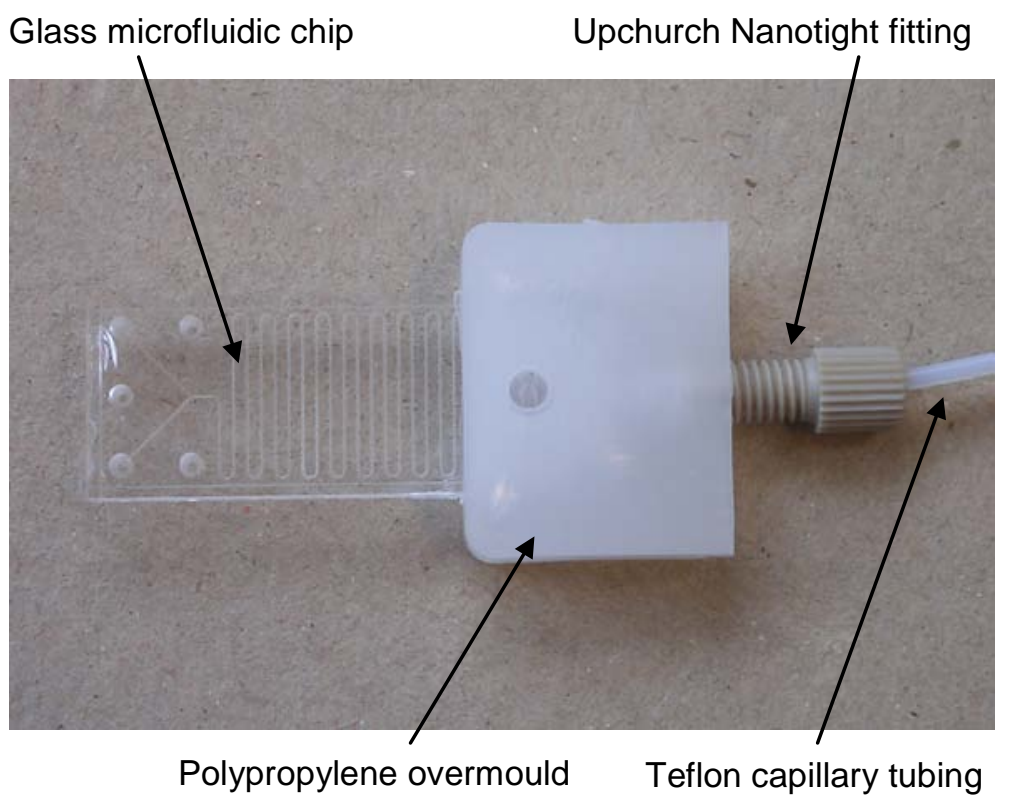

Figure 4. Glass microfluidic chip connected to Teflon capillary tubing via a polypropylene overmould

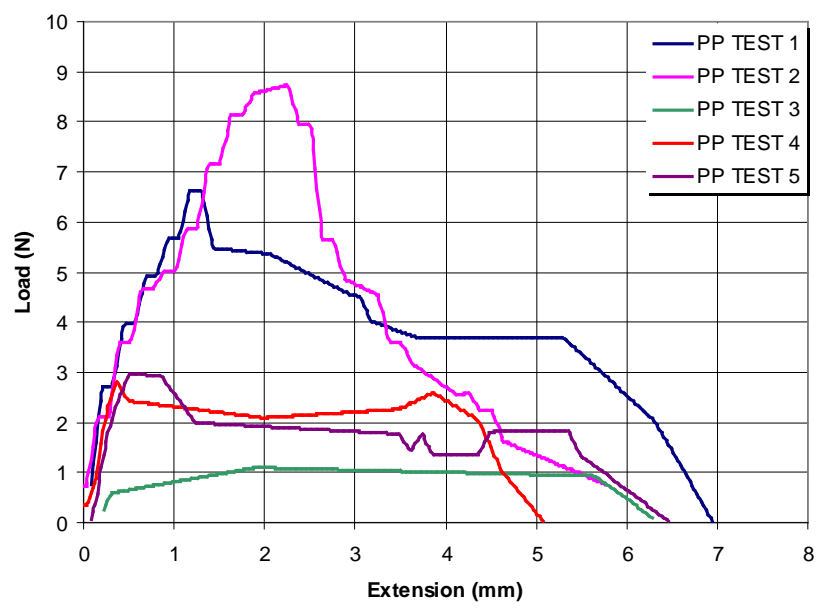

Figure 5. Capillary tubing pull-out test results, (a) with a polypropylene moulding, (b) with an Upchurch Nanoport.

In another characterisation test the force required to pull the capillary tubing out of the compression assembly was measured. The test was performed using a polypropylene moulding without a glass insert to simplify the test setup. The results were compared to pull out measurements using a Upchurch Nanoport fitting instead of the moulding as the male part of the compression assembly. For each test the capillary was inserted and the compression nut screwed in $3 \frac{1}{2}$ turns. This was enough to make the nut fingertight for both the Nanoport and the moulding. The tube was then pulled out and the same parts reassembled for the next test. The force measurements are shown in Figure 5. The maximum load seen in five tests with the moulding was $8.8 \mathrm{~N}$ and with the Nanoport 7.5N. The results are broadly similar, demonstrating that the wall 
thickness of the overmould is sufficient to allow the compression fitting to work as designed.

\section{Induction Heating for Sealing of Microfluidic Devices}

Low frequency induction heating (LFIH) is well established in the steel industry for hardening, melting, soldering, welding, and annealing, and increasingly is finding application in other areas like heating fillings in dental medicine, and bottle cap sealing. The advantages of this technique are manifold. The process is energy efficient, and because heating is rapid and no chamber is required it is capable of high production rates. To join plastics a susceptor placed at the joining interface can be used - a metal component that absorbs energy from the induction field. In this application LFIH has the further advantage of delivering heat directly to the joining interface, reducing the amount of heat distortion in the part.

The results of feasibility trials on the use of LFIH to lid polymer microfluidic devices have been reported (Knauf et al 2008). $2 \mathrm{~mm}$ thick $25 \mathrm{~mm} \times 25 \mathrm{~mm}$ in area PMMA plates were joined by placing a thin film susceptor between the plates, clamping and heating in an induction field. Visible melting of the polymer and strong joints were formed in seconds with use of a $7.5 \mu \mathrm{m}$ nickel foil as susceptor, see Figure 6. The joints were pressure tested with air to 5.9 bar without failing.
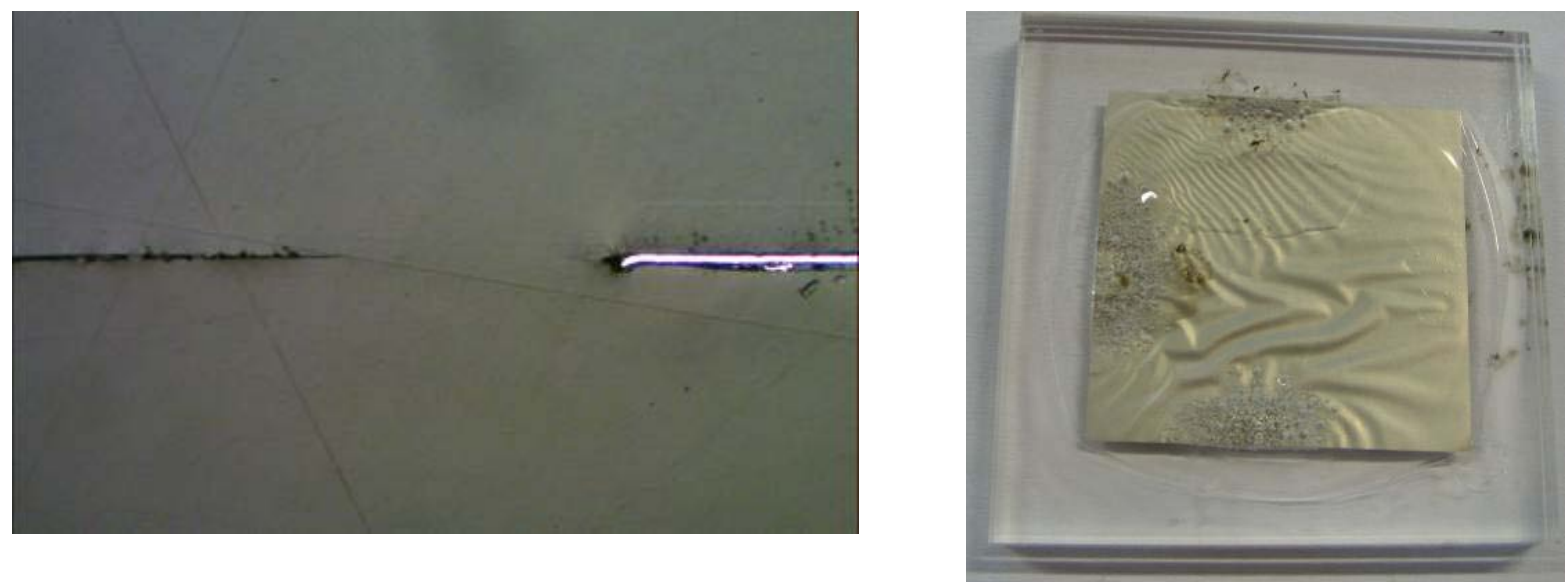

Figure 6. (a) $2 \mathrm{~mm}$ thick PMMA plates joined using a $7.5 \mu \mathrm{m}$ nickel foil susceptor, (b) cross section of joint.

The plated layer should be as thin as possible. In the feasibility trials a $5 \mu \mathrm{m}$ plated nickel coating was also found to heat sufficiently to melt the PMMA. However, because the layer covered the whole of the surface of one of the plates no plasticplastic bond was formed, and the plastic-metal bond was found to be weak. A 50100nm evaporated nickel coating heated but not sufficiently to melt the PMMA. While ferromagnetic materials such as nickel have the strongest coupling to the induction field, a $5 \mu \mathrm{m}$ thick non-ferromagnetic coating of sputtered aluminium was also found to heat rapidly. Current work concentrates on optimising the LFIH process parameters to achieve bonding with minimum heat affected zone (HAZ) thickness. The use of conductive paste printed into channels on the surface of a chip to act as a susceptor is also being trialled. A preliminary result using a silver paste yielded a HAZ of about $90 \mu \mathrm{m}$ thickness. 


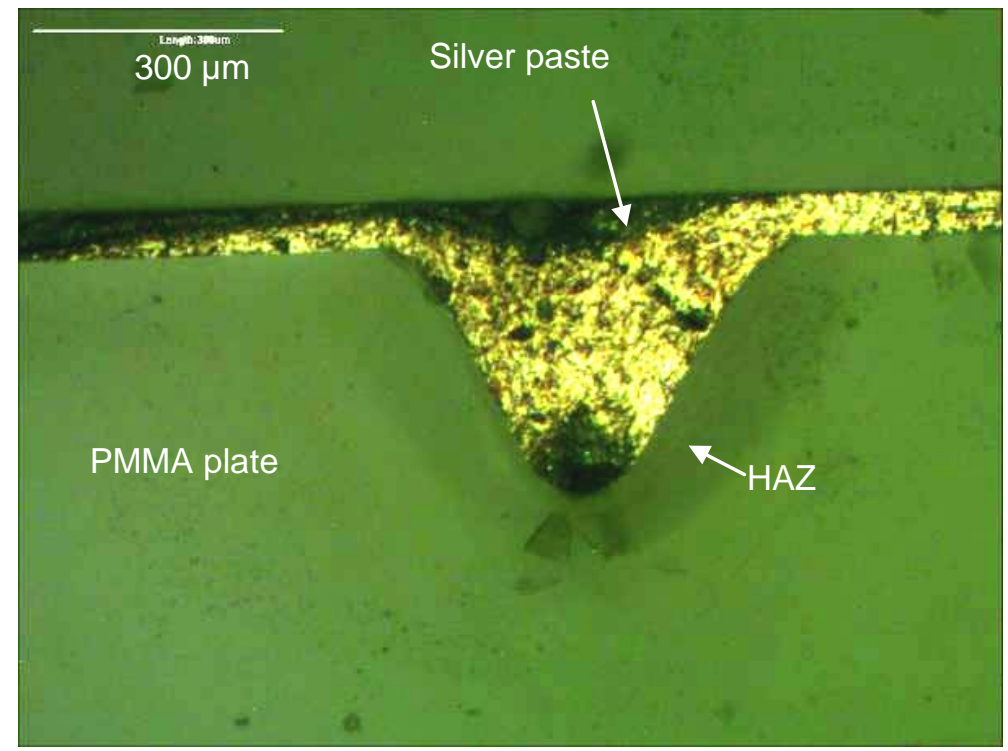

Figure 7. Cross-section of silver paste filled into a groove on the surface of a PMMA plate and heated using LFIH, viewed using polarisers. A heat affected zone of about $90 \mu \mathrm{m}$ thickness can be seen.

\section{Discussion}

In a manufacturing process depositing a continuous susceptor layer over the surface of a lid for a microfluidic device, Figure 7(a), would be the simplest to achieve. However this would have the drawback that the susceptor material would be wetted by the working fluid in use. In addition as described in Section 4 above the metal-topolymer bond achieved by induction heating was found to be weak. Thus it is envisaged that in application a patterned susceptor would be used following the edges of the microfluidic channels, as illustrated in Figure 7(b). This would allow plasticplastic bonds to be formed and the complete encapsulation of the susceptor material.

Figure 8. Concepts for susceptor patterning: (a) continuous layer, (b) pattern following the edges of the microfluidic channels (Knauf et al. 2008)

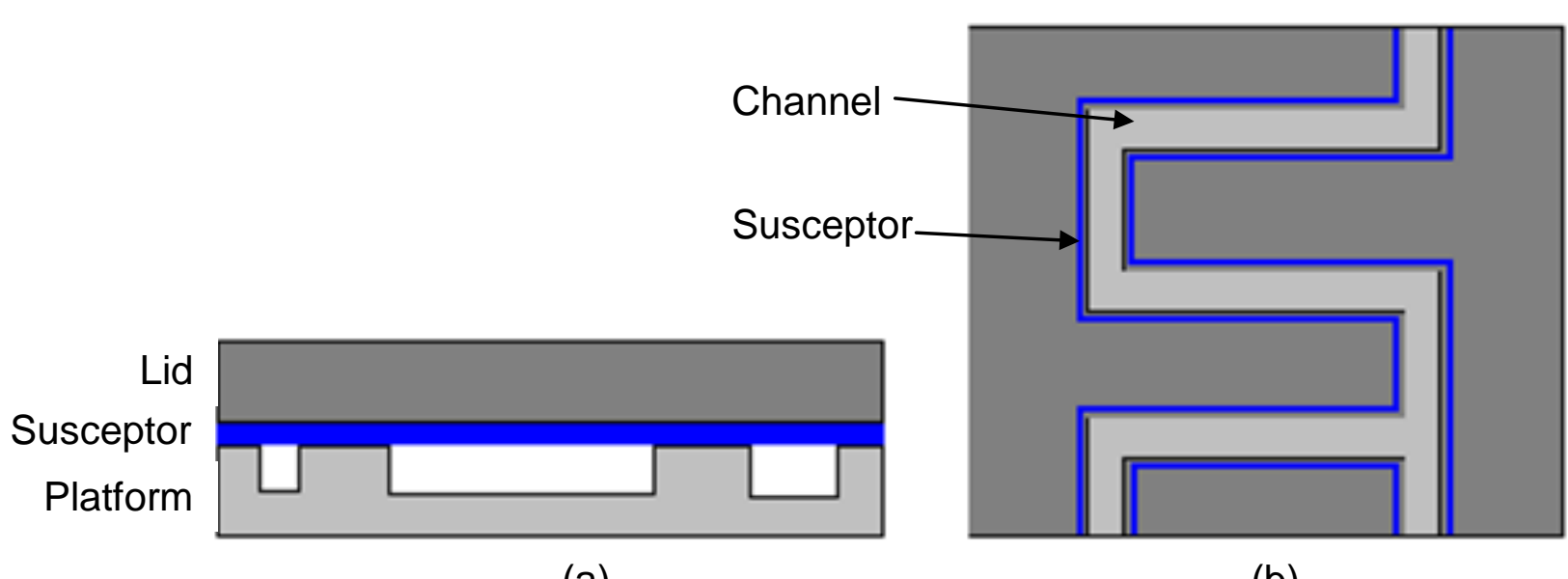

(a)

(b) 
An extension of LFIH would be to join tubing to the microfluidic device using a suitably designed metal ferrule as susceptor. . The spatial selectivity of induction heating offers the prospect that in a mature process lid sealing, and connectorisation could be done simultaneously. In addition a stack of polymer layers could be bonded simultaneously to create a multi-layer microfluidic device. A possible extension of both the overmoulding and LFIH methods is to combine them to create a process for integration of microfluidics as illustrated in Figure 8. Separate microfluidic elements, for example a channel network and a flow-through sensor would be embedded in an overmould. The surface of the overmould would itself be structured with an interconnecting fluidic channel network. Induction heating would then be used to seal the interconnection network. The integrated module would thus be created in only two manufacturing steps.

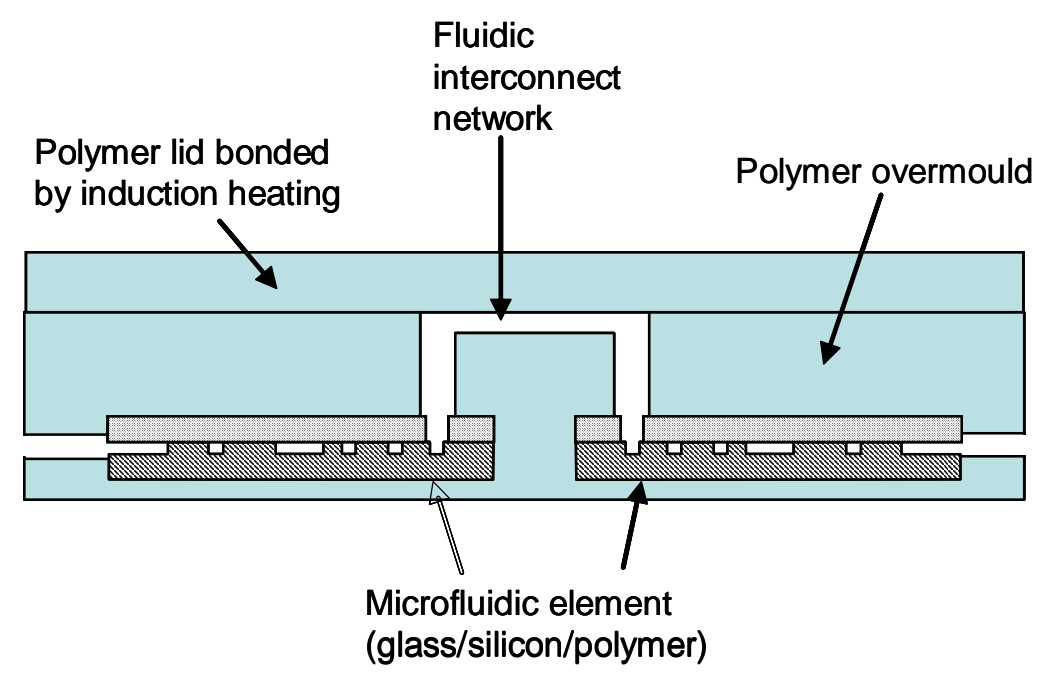

Figure 9. A concept for manufacture-friendly integration of microfluidic elements

\section{Conclusions}

Microfluidic chips have until now been produced in relatively small numbers. If the high growth rates which have been predicted for the sector are to be achieved, technical barriers to product development and manufacture in high volumes will have to be overcome. Packaging issues have been highlighted as major challenge, in particular fluidic interconnection, and lid sealing of polymer microfluidic devices. In addition prototyping and bio-molecule compatibility have been identified as challenges for polymer microfluidics, which will be the material of choice for low cost high volume manufacture.

Development work at Loughborough University to address packaging issues has been reviewed and updated. This includes an overmoulding process to create a connectorised module from a microfluidic chip. Feasibility has been shown for glass microfluidics using direct adhesion between the overmould material and the glass surface as the fluid seal principle. The seal has been demonstrated to survive water pressurisation for 116 hours. A second design to make connection to a Teflon capillary tube using an Upchurch fitting has also been implemented and tested successfully.

Trials of a low frequency induction heating (LFIH) process to assemble the lid to polymer microfluidic channel networks have also been described. Acrylic plates have 
been joined using a thin $(<10 \mu \mathrm{m})$ nickel susceptor providing a fluid seal that withstood a pressure of $590 \mathrm{kPa}$. A possible extension of both the overmoulding and LFIH methods is to combine them to create a process for integration of multiple microfluidic elements within a polymer block.

\section{Acknowledgements}

The authors would like to thank Chandrahas Parmar for the preparation and testing of the polypropylene overmoulds. This work forms part of the 3D Mintegration Grand Challenge, EPSRC grant reference: EP/C534212/1.

\section{Reference List}

Becker, H. and Gaertner, C. ( 2008), "Polymer Microfabrication Technologies for Microfluidic Systems.", Analytical and Bioanalytical Chemistry, 390,1,pp89111.

Bourne, M. (2005). "2005 may be a momentous year for MEMS, or maybe just a momentum-building year", 21 Jan, Available at: http://www.smalltimes.com/print_doc.cfm?doc_id=8635. (Accessed 24 Jan 2005).

Ellis, M. (2009), "Plastic Diagnostic Lab-on-a-Chip.", Materials World, 17, 1, pp

Freemantle, M. (1999), "Downsizing Chemistry.", Chemical Engineering News, February 22, pp 27-36

Grujicic, M., Sellappan, V., Omar, M.A., Seyr, N., Obieglo, A, Erdmann, M. and Holzleitner, J. (2008), " An Overview of the Polymer-to-Metal DirectAdhesion Hybrid Technologies for Load-Bearing Automotive Components." , Journal of Materials Processing Technology, 197, 1-3, pp 363-73.

Ho, C.M. (2001), "Fluidics - the Link Between Micro and Nano Sciences and Technologies.", in 14th Ieee International Conference on Micro Electro Mechanical Systems, Interlaken, Switzerland, Jan 21-25 2001, pp 375-384

Hupert, M.L., Witek, M.A., Wang, Y., Mitchell, M.W., Liu, Y., Bejat, Y., Nikitopoulos, D.E., Goettert, J., Murphy, M.C. and Soper, S.A. ( 2003), "Polymer Based Microfluidic Devices for Biomedical Applications." in Microfluidics, BioMEMS and Medical Microsystems, Holger Becker and Peter Wojas (Eds), pp. 52-64

Kalaugher, L. (2004), "Nanotube sensors monitor blood glucose levels",16 Dec, Available at: http://nanotechweb.org/articles/news/3/12/11 (Accessed 20 Dec 2004).

Knauf, B. J., Webb, D.P., Liu,C, and Conway., P.P (2008), "Packaging of Polymer Based Microfluidic Systems Using Low Frequency Induction Heating (LFIH).", in International Conference on Electronic Packaging Technology \& High Density Packaging, 2008 ICEPT-HDP 2008,Shanghai, PRC, Jul 28-31, pp 1-6

Manz, A., Graber, N. and Widmer, H.M. (1990), " Miniaturized Total Chemical 
Analysis Systems: A Novel Concept for Chemical Sensing.", Sensors and Actuators B: Chemical, 1, 1-6, pp 244-48.

Mounier, E. and Michel, P. (2003), "Technologies and Markets Trends in Biochips and Microfluidic Chips.", in Lab Automation 2003, Palm Springs, USA, Feb 1-5, pp 1-7

PRIME Faraday Technology Watch. (2002), An Introduction to MEMS, PRIME Faraday Partnership, Loughborough, United Kingdom., Available online: http://www.integratedproductsktn.org.uk/

Schena, M. (Ed) (2000), Microarray Biochip Technology, Eaton Publishing, Natick, USA

Sia S.K. and Kricka L.J. (2008), "Microfluidics and Point-of-Care Testing.", Lab On A Chip , 8, 12, pp 1982-83.

Verpoorte, E. and De Rooij, N.F. (2003), "Microfluidics Meets MEMS." , Proceedings of the IEEE, 91, 6, pp 930-953.

Webb, D.P., Hutt, D.A., Hopkinson, N., Conway, P.P., and Palmer, P.J (2009), "Packaging of Microfluidic Devices for Fluid Interconnection Using Thermoplastics." Journal of Microelectromechanical Systems, 18, 2, pp 354362

Webb, D.P., Hutt, D.A., Hopkinson, N., Palmer, P.J. and Conway, P.P. ( 2006), "Integration and Packaging of Microsystems by Polymer Overmoulding.", in 1st Electronics Systemintegration Technology Conference, Dresden, Germany, 5-7 Sept, pp 567-74. 


\section{List of Figures}

Figure 1. A microfluidic assembly consisting of a fluidic substrate containing the microfluidic channels, a capping layer sealing the channels, fluidic input/output ports and a structure to support fluidic interconnection such as connectors for tubing.

Figure 2. Concept of moulded interconnect to an embedded microfluidic device.

Figure 3. Design of moulding to allow connection of a capillary tube to a microfluidic device using a screw thread compression fitting.

Figure 4. Glass microfluidic chip connected to Teflon capillary tubing via a polypropylene overmould

Figure 5. Capillary tubing pull-out test results, (a) with a polypropylene moulding, (b) with an Upchurch Nanoport.

Figure 6. (a) $2 \mathrm{~mm}$ thick PMMA plates joined using a $7.5 \mu \mathrm{m}$ nickel foil susceptor, (b) cross section of joint.

Figure 7. Cross-section of silver paste filled into a groove on the surface of a PMMA plate and heated using LFIH, viewed using polarisers. A heat affected zone of about $90 \mu \mathrm{m}$ thickness can be seen.

Figure 8. Concepts for susceptor patterning: (a) continuous layer, (b) pattern following the edges of the microfluidic channels (Knauf et al. 2008)

Figure 9. A concept for manufacture-friendly integration of microfluidic elements 